\title{
LECTURE
}

\section{Why Do Japan's Advanced Medical Treatments Never Get Ahead?}

\author{
Nobuyo Hatanaka, ${ }^{1}$ Shinichi Matsumoto, ${ }^{1,2}$ Yuji Tanaka ${ }^{1}$ and Masahiro Kami ${ }^{1}$ \\ ${ }^{1}$ Division of Social Communication System for Advanced Clinical Research, \\ the Institute of Medical Science, the University of Tokyo, Tokyo, Japan \\ ${ }^{2}$ Baylor Research Institute Fort Worth Campus, Texas, USA
}

(Received for publication on September 2, 2009)

(Revised for publication on November, 4, 2009)

(Accepted for publication on November 19, 2009)

\begin{abstract}
Islet cell transplantation is a minimally invasive procedure which effectively controls blood glucose level for diabetic patients but is considered as experimental. After islet transplantation, type 1 diabetic patients could become insulin free with stable glycemic control. But for long term effects, only stable glycemic control was maintained and not insulin free status. In 2004 Kyoto University performed the first Japanese islet cell transplant using non-heart beating donor. Of note, due to the lack of cadaveric donors in Japan, the same group performed the world's first successful case of living donor islet transplantation in 2005. Both patients achieved transit insulin-independence; however excellent glycemic control was able to be maintained for a prolonged period. Even though the series of islet transplants at Kyoto University showed promising results, the leading scientist did not continue his research in Japan. This was because it is extremely difficult to implement newly developed treatment as a standard therapy in Japan. (Keio J Med 59 (2) : 46-51, June 2010)
\end{abstract}

Keywords: islet transplantation, type 1 diabetes, bio-artificial islet

\section{Introduction}

In Japan, in order for new medical technology to be approved by the Drugs, Cosmetics and Medical Instruments Act (DCMIA) to obtain approval as standard treatment, the Highly Advanced Medical Treatment System (HAMTS) has been widely used by medical doctors. However, in September 2006, the HAMTS was abolished resulting in closing the way for new technologies to become standard treatments for medical doctors in Japan. The new technology for islet cell transplantation, which is aimed at treating type 1 diabetes mellitus, was affected also.

Due to the fact that organs from brain-dead donors are not available for islet cell transplantation in Japan, organs from non-heart-beating donors (NHBDs) were used. ${ }^{1}$ Islet cell transplantation from NHBDs is extremely difficult, but with excellent pancreatic islet isolation techniques, it was rendered possible. These islet isolation techniques have been highly acclaimed all over the world, and the success of islet cell transplantation using NHBDs became a leading achievement in terms of the success rate of pancreatic islet isolation. ${ }^{2}$ These islet isolation techniques also enabled to perform "living donor islet cell transplantation", whose validity for islet isolation needed confirmation for the first time in the world. ${ }^{3,4}$ Even though they possessed high-level technology which has earned evaluations all over the world, Japanese researchers did not adopt it as standard treatment, despite the fact that patients were in need of new medical treatments. In this article, we describe the reasons why re-importation from the United States is needed to be done.

Presented at the 1631st meeting of The Keio Medical Society in Tokyo, July 10, 2009.

Reprint requests to: Shinichi Matsumoto, MD, PhD, Baylor Research Institute Fort Worth Campus, $14008^{\text {th }}$ avenue Fort Worth, Texas 76104, USA, E-mail: shinichm@baylorhealth.edu 


\section{Transplantation for Insulin Dependent Diabetic Patients}

Insulin-dependent diabetes mellitus such as type 1 diabetes is a condition in which the insulin-producing cells (beta cells) are lacking, and supply of insulin from outside the body is necessary to maintain life. Unlike noninsulin-dependent diabetic conditions such as type 2 diabetes, in type 1, improvement of symptoms cannot be achieved by changing lifestyle habits alone. For insulindependent diabetes, controlling the blood glucose level is sometimes difficult even with intensive insulin therapy. The disease often takes on the form of hypoglycemia unawareness or severe hypoglycemia in which patients cannot detect the low blood glucose levels that could result in dangerous coma and seizure. Hypoglycemia unawareness and severe hypoglycemia are not the types of hypoglycemia that can be prevented by patients themselves. Hypoglycemia unawareness is also one of the factors which limit the patients from going out alone. As a result, the patient's quality of life (QOL) will severely decline. Under such circumstances, transplantation of beta cells will be considered as the next step of treatment. There are two ways of possible transplantation of beta cells. One is pancreas transplantation which can be provided as a standard treatment covered by medical insurance. The other is islet cell transplantation which is at the level of experimental treatment, and is provided at the patient's own expense or at the cost of research funds. In either case, the purpose of the treatment is to stabilize the blood glucose levels and to improve the QOL of the patients.

There are risks involved in pancreas transplantation performed under general anesthesia as major abdominal surgery. Therefore, pancreas transplants have predominantly been performed simultaneously with kidney transplants for which the advantages outweigh the risks. If this simultaneous pancreas and kidney transplant is successful, then unstable blood glucose level and dialysis can be solved with a single surgery. Many diabetic patients also suffer from vascular complications. When patients are anxious about their physical conditions, in order to choose pancreas transplantation, they should be well informed and prepared. If the patient's body rejects the newly transplanted organ, the patient must undergo another surgery to remove the transplanted pancreas.

In contrast, in the case of islet cell transplantation, the procedure can be more easily performed by packing islet cells into transfusion bags and performing transplantation through an intravenous route. A catheter is inserted percutaneously into the portal vein under local anesthesia, and transplantation is performed through the catheter while paying attention to the portal blood pressure. ${ }^{5}$ Transplantation itself is very simple and safe. In addition, only islet cells will be spontaneously eliminated when they are rejected. For these reasons, when patients are anxious about their physical conditions, they have the option to choose islet transplantation over the highly invasive surgical procedure.

Current major issues of islet transplantation are, 1) low success rate of islet isolation, 2) requirement of multiple donors for achieving insulin-free status, and 3) difficulty to maintain long term insulin independence. If we are able to overcome these major issues, islet transplantation could become the standard therapy. However, even if islet transplantation becomes the standard therapy, there is still the major issue of organ donor shortages.

\section{Islet Cell Transplantation in Japan}

Japan's first clinical islet cell transplant was performed at Kyoto University in 2004. ${ }^{1}$ All over the world islet cell transplantation is performed by transplanting islet cells from the pancreas of brain-dead donors. ${ }^{5}$ In Japan however, islet cell transplantation is performed by using the pancreas of non-heart-beating donors (NHBD). Compared to that provided by brain-dead donors, pancreata from NHBDs are in marginal condition, making islet isolation from NHBDs difficult. However, the Kyoto University group demonstrated that their islet transplant results with NHBDs were almost comparable to the results with brain-dead donors. ${ }^{2}$

The process of islet cell isolation is the keystone of islet cell transplantation. Islet transplantation is consisted of four following processes; 1) retrieval of the pancreas, 2 ) preservation of the pancreas, 3 ) isolation of the pancreatic islets, and 4) purification of the pancreatic islets. ${ }^{6}$ Many researchers are examining the pancreatic islet isolation process. However, the Kyoto University group added modifications to these processes. The technique including these processes was later called the Kyoto Method. The Kyoto Method became the leading method in the world in terms of islet cell transplantation due to the high success rate of islet isolation.

Islet cell transplantation was at the level of experimental treatment not only in Japan but in the United States also, and is aimed to become the standard of medical care worldwide. Japan's data on the success rate of islet isolation for islet cell transplantation showed highest results in the world, and was praised as the birth of new medical technology.

\section{Japanese Patients' Perspective of Islet Cell Transplantation}

Pancreas transplantation and islet cell transplantation are both beta cell replacement therapies for type 1 diabetes mellitus, and knowing the patients' actual expectations for the treatment is important in conducting future research. For this reason we conducted a survey to understand patients' expectations. ${ }^{7}$

From May to September of 2008 in a survey involving 
105 type 1 diabetes patients, we conducted an investigation on the patients' inclination in terms of treatment of the disease. For each question, we prepared five choices: "strongly agree", "agree", "somewhat agree", "disagree", and "strongly disagree."

First, we asked which of the new treatments the patients wanted to undergo in order to be freed from the disease and what they expected from it. Among the new treatments were transplantation and bio-artificial endocrine pancreatic islet transplantation.

To the question "Would you want to receive a new treatment if it frees you from the disease?" $41 \%$ responded "strongly agree", $25 \%$ responded "agree", $27 \%$ responded "somewhat agree", 4\% answered "disagree", and 3\% answered "strongly disagree". From these answers, negative answers regarding new treatments amounted to $7 \%$, while positive answers were as many as $93 \%$ (Fig. 1A). Next, to the question "Do you think that transplantation is the alternative treatment to insulin therapy?" $22 \%$ responded "strongly agree", $24 \%$ responded "agree", $25 \%$ responded "somewhat agree", $19 \%$ answered "disagree", $5 \%$ answered "strongly disagree", and 5\% did not give any answer. From these results, $71 \%$ were positive opinions in favor of medical transplantation (Fig. 1B). Finally, to the question "Would you want to be treated with a preparation using swine cells if it is effective?" $14 \%$ responded "strongly agree", $23 \%$ responded "agree", $24 \%$ responded "somewhat agree", 19\% answered "disagree", 16\% answered "strongly disagree", and 4\% did not give any answer. From these results, $61 \%$ of the subjects gave positive answers to new treatments if the treatments are effective (Fig. 1C). Due to the limited number of organ donors, allogenic islet cell transplantation cannot be made available to all type 1 diabetic patients. Therefore, it is necessary to seek different sources, and the most promising ones are swine pancreatic islets. Nowadays, research on the use of bio-artificial islet transplantation with swine pancreatic islets is being conducted worldwide, and their use in clinical applications is already underway. ${ }^{8} \mathrm{How}-$ ever, for the future encouragement of bio-artificial islet transplantation, it is important to know the percentage of transplant recipient patients who would actually accept cells from a different species. From the results of this survey, the fact that "the majority of the patients thought positively about accepting" is worth mentioning. These results can also suggest that type 1 diabetic patients have high expectations regarding new treatments.

When type 1 diabetic patients come to the hospital at the onset of the disease for the first time, it is explained that insulin therapy must be continued for a lifetime. In other words, type 1 diabetic patients live their entire lives with the disease, which may bring an enormous mental burden to the patients. Therefore, we analyzed the patients' anxiety and fear of diabetes. As a result to the question "Do you feel anxiety and fear for complica- tions?" 37\% responded "strongly agree", 29\% responded "agree", and $28 \%$ responded "somewhat agree". Therefore, those who felt fear and anxiety of complications accounted for $94 \%$ (Fig. 2A). To the question "Does type 1 diabetes mellitus cause difficulties in everyday life?" 28\% responded "strongly agree", $29 \%$ responded "agree", and 24\% responded "somewhat agree". Therefore, $81 \%$ of the respondents experienced some impairment (Fig. 2B). In the long-term fight against this illness, patients have a very strong fear of developing complications and want to recover completely if possible. Therefore, we can assume that their expectations regarding new treatments are high.

\section{From Experimental Medicines to Standard Medical Care}

In Japan, several systems are offered as a way for experimental treatments to become standard medical care.

First, Highly Advanced Medical Technology (HAMTS) had been widely used by medical doctors. Doctors in Japan can initiate new medical treatments under approval of the Institutional Review Board. After completing cases which could confirm the usefulness and safety of a new treatment, doctors could submit their applications through HAMTS. Subsequently, a review will be conducted by the Central Social Insurance Medical Council (CSIMC) of the Ministry of Health, Labour, and Welfare (MHLW) through an extensive medical audit meeting to examine the technology. When safety and efficacy are confirmed, subsequently, suitability for insurance coverage is discussed at a meeting specializing in advanced medical care that examines insurance at the CSIMC. When the treatment is recognized as HAMT, then the treatment can be provided to patients from health insurance combined with a co-payment by the patients. Further research is conducted and data aiming at approval as standard medical care can be collected. However, in accordance with the partial revision of the Health Insurance Act on October 1, 2006, the HAMTS and the Leading Edge Health Care System (LEHCS) were merged, and the advanced health care system was reorganized into a new system.

The second system is the Advanced Medical System (AMS). Unlike the HAMTS, the condition for submission of notification is that the medical technology uses medical supplies and medical equipment which have received approval from the DCMIA, or that the medical technology is being examined for introduction into the insurance system. A notification is sent to the MHLW where it is received by internal subdivisions in charge of the insurance department, where its adaptation for combination with insurance is judged by a conference of experts on advanced medical care. Subsequently, the effectuation (establishment criteria) of advanced medical care will be set, and its combination as healthcare services 
A

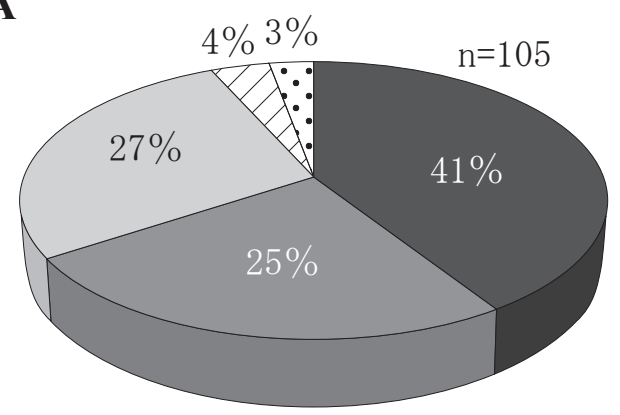

B

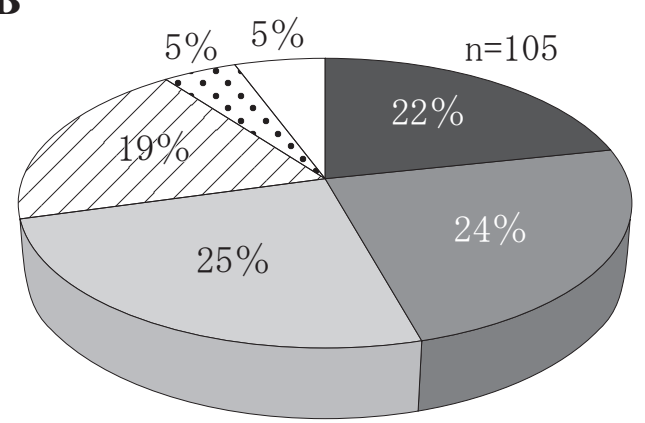

C

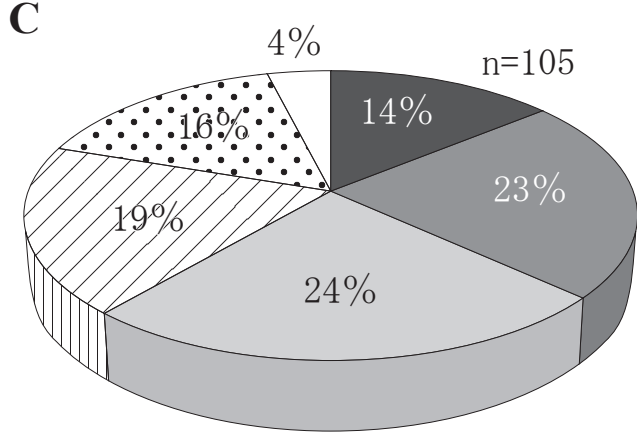

Strongly agree

$\square$ Agree

$\square$ Some what agree

$\square$ Disagree

- Strongly disagree

$\square$ Unanswered

Fig. 1 Patients' opinions of new treatment (A), transplantation (B), and xeno-transplantation (C) for the treatment of diabetes.

A $4 \% 2 \%$

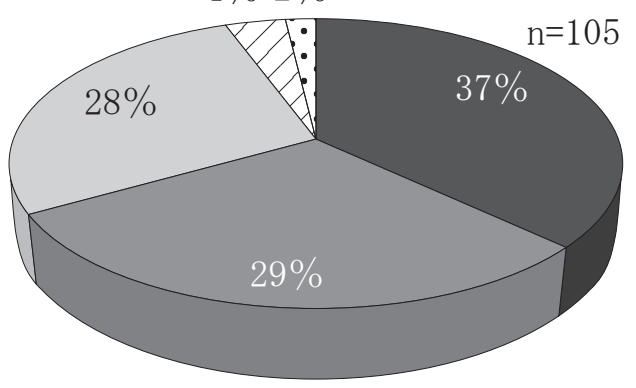

B

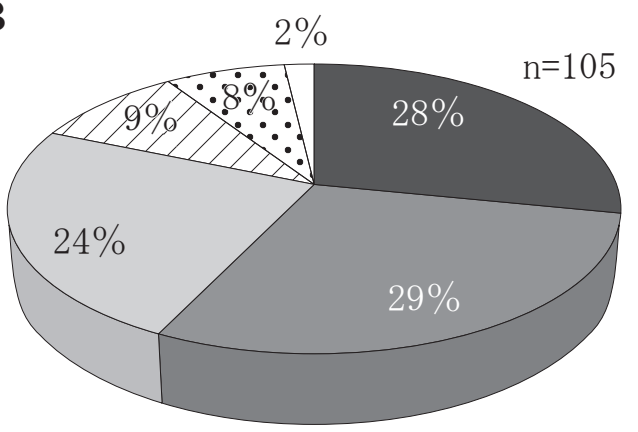

Strongly agree

Agree

$\square$ Somewhat agree

$\square$ Disagree

- Strongly disagree

$\square$ Unanswered

Fig. 2 Patients' opinions about anxiety and fear (A) and difficulties (B) related to diabetes. 
provided by health insurance is made possible. Until October 2006 this AMS was called the "Leading Edge Health Care System," and after October 2006 it was integrated with the HAMTS, and its name has also changed. This system could not be applied for islet transplantation because islet transplantation uses unapproved drugs.

The third system is the Evaluation of Advanced Healthcare System (EAHS). This system was founded in April 2008 and had contents similar to the HAMTS which had been abolished. It deals with medical technologies using medical supplies/medical equipment not yet approved and indicated by the DCMIA, for which applications are sent to the MHLW as high level healthcare. Safety and efficacy are confirmed by a conference within the EAHS at the Health Policy Bureau within the internal subdivisions of the MHLW, and then the adequacy of its combination with insurance is determined by the conference of experts on advanced medical care. As a result, extensive medical care is determined as advanced medical care, and the combination as healthcare services provided by health insurance is made possible. This system is available for islet transplantation nowadays.

\section{The Researchers' Dilemma after the Abolition of the HAMTS}

With the abolition of the HAMTS in 2006, medical doctors lost the way to implement innovative treatments as standard medical care, and subsequent plans had to be established. Other than the HAMTS, clinical trials sponsored by medical doctors have been under consideration.

For most clinical trials conducted by pharmaceutical companies, research funds are calculated and spent only if sufficient amount of profits can be expected in the future. For this reason, Japanese pharmaceutical companies rarely spend research funds for treatments like islet cell transplantation in which the number of targeted patients is small. Although islet cell transplantation developed in Japan was a leading medical technology in the world, there was hardly a way to make it standard treatment in Japan.

Until recently, Japan has been promoting healthcare in the form of medical care imported from Western countries. For this reason, only certain exceptional new medical treatments were implemented into standard medical care. Living donor liver transplantation was one such exception. And it was through the HAMTS that it was able to become standard healthcare. In order to establish islet cell transplantation as standard medical care, establishment as medical care in the United States was aimed as a shortcut to re-import it later. There were no considerations to conduct clinical trials which would have required a new medical system and financial support.

\section{Do New Technologies in Japan Come Through the United States?}

The low success rate of islet isolation, the necessity of multiple donors for achieving insulin-independence, and the difficulty of long-term insulin-free independence are three major issues for islet transplantation.

In the United States, data regarding islet cell transplantation have improved after introduction of the Kyoto Method. Success rate in isolating transplantable islets from a donor's pancreas has increased to approximately $100 \%,{ }^{9}$ while with the University of Alberta, ${ }^{10}$ the University of Miami, ${ }^{11}$ and the University of Minnesota, ${ }^{12}$ known leaders in terms of islet cell transplantation, the rates were reported to be about $40 \%$.

The number of pancreatic islets in a healthy human being is approximately 1 million. It has been reported that using the current standard method of islet isolation, an average number of 300,000 pancreatic islets can be isolated from one pancreas. With the Kyoto Method, an average of 700,000 pancreatic islets can be isolated. ${ }^{13}$ Therefore, insulin-independence may be gained through transplantation of islet cells isolated from one pancreas. In fact, with the latest islet cell transplantation protocol implemented by Baylor Regional Transplant Institute, blood glucose level stabilization and withdrawal from insulin injections were achieved after a single donor islet transplant. ${ }^{14}$

Our first islet cell transplant in the United States was conducted in February of 2007. We were able to provide high quality and quantity of islets to the patient, and to date the patients remain insulin-free, and was able to maintain excellent glycemic for more than two years. ${ }^{15}$ These are promising results for the maintenance of longterm insulin-free independence.

With the introduction of new islet isolation technologies, the once major issues of islet transplantation seem to be resolved. We are currently preparing to apply for FDA approval to promote our islet transplantation as the standard therapy in the United States. Once this standard of therapy is approved, we should be ready for re-importation of islet transplantation into Japan.

\section{Conclusion}

Japan has a history of introducing new medical care from foreign countries because its national system for the establishment of standard treatments is weak. Furthermore, since the HAMTS has been abolished, it has become difficult for new medical treatments to become standard treatments. Re-importation from Europe and the United States has been considered to be a shortcut for the development of new medical care, and even pharmaceutical companies perform clinical trials in Europe and the United States. As globalization progresses, researchers look for places to exercise the maximum of 
their own capabilities, and aim to establish new medical care.

Ironically, Japan has been conducting research in order to develop technologies that can be attainable worldwide, yet has abandoned their establishment in the final stages.

Is there a way to improve the current situation in which we are forced to aim establishing technologies in the Western countries? The earnest desire of Japanese patients waiting for new medical care is for Japanese researchers to aim at the establishment of medical care in Japan without having to go overseas.

We hope that the EAHS will be a shortcut for the establishment of new medical care. For this, we should continuously improve the EAHS system to make it more effective. Medical doctors and Japanese government should work together to improve this system.

Additionally, Japan should also consider building an environment in which researchers can devote themselves to their research without losing motivation for establishing new medical care.

\section{Acknowledgements}

This study was supported in part by a grant from the Health Labour Sciences Research Grant for Research on Basic Researches from the Japanese Ministry of Health, Labour and Welfare (H19-Trans-General-004: Establishment of islet cell transplantation as the result of translational research).

\section{References}

1. Matsumoto S, Okitsu T, Iwanaga Y, et al: Successful islet transplantation from nonheartbeating donor pancreata using modified Ricordi islet isolation method. Transplantation 2006; 82: 460465.

2. Liu X, Matsumoto S, Okitsu T, et al: Analysis of donor-and isolation-related variables from non-heart-beating donors (NHBDs) using the Kyoto islet isolation method. Cell Transplant 2008; 17: 649-656.

3. Matsumoto S, Okitsu T, Iwanaga Y, et al: Insulin independence after living-donor distal pancreatectomy and islet allotransplantation. Lancet $2005 ; 365$ : 1642-1644.

4. Matsumoto S, Okitsu T, Iwanaga Y, et al: Follow-up study of the first successful living donor islet transplantation. Transplantation 2006; 82: 1629-1633.

5. Shapiro AM, Lakey JR, Ryan EA, Korbutt GS, Toth E, Warnock GL, Kneteman NM, Rajotte RV: Islet transplantation in seven patients with type 1 diabetes mellitus using a glucocorticoid-free immunosuppressive regimen. N Engl J Med 2000; 343: 230-238.

6. Matsumoto S, Noguchi H, Naziruddin B, et al: Improvement of pancreatic islet cell isolation for transplantation. Proc (Bayl Univ Med Cent) 2007; 20: 357-362.

7. Hatanaka N, Takita M, Yamaguchi T, Kami M, Matsumoto S: Interests in beta-cell replacement therapies among Japanese patients with type 1 diabetes. Diabetes Res Clin Pract 2010; Epub ahead of print.

8. Valdes-Gonzalez RA, White DJ, Dorantes LM, et al: Three-yr follow-up of a type 1 diabetes mellitus patients with an islet xenotransplant. Clin Transplant 2007; 21: 352-357.

9. Matsumoto S, Noguchi H, Shimoda M, et al: Seven consecutive success of clinical islet transplantation with pancreatic ductal injection. Cell Transplant (in press).

10. Kin T, Mirbolooki M, Salehi P, et al: Islet isolation and transplantation outcomes of pancreas preserved with University of Wisconsin solution versus two-layer method using preoxygenated perfluorocarbon. Transplantation 2006; 82: 1286-1290.

11. Ichii H, Pileggi A, Molano RD, et al: Rescue purification maximizes the use of human islet preparations for transplantation. Am J Transplant 2005; 5: 21-30.

12. Matsumoto I, Sawada T, Nakano M, et al: Improvement in islet yield from obese donors for human islet transplants. Transplantation 2004; 78: 880-885.

13. Matsumoto S, Noguchi H, Hatanaka N, et al: Estimation of Donor Usability for Islet Transplantation in the United States with the Kyoto Islet Isolation Method. Cell Transplant 2009; 18: 549-562.

14. Matsumoto S, Noguchi H, Takita M, et al: ET-Kyoto ductal injection and density adjusted purification combined with potent antiinflammatory strategy facilitated single donor islet transplantation. Xenotransplantation 2009; 16: 396-397.

15. Matsumoto S, Noguchi H, Takita M, et al: Super high dose islet transplantation is associated with high SUITO index and prolonged insulin independence. Xenotransplantation 2009; 16: 397. 